\title{
SynTRACE-21: Synthesis of Transient Climate Evolution of the last 21,000 years
}

\author{
Zhengyu Liu', B.L. Otto-Bliesner², P.U. Clark³ ${ }^{3}$ J. Lynch-Stieglitz ${ }^{4}$ and J.M. Russell ${ }^{5}$
}

SynTRACE-21 initiated a comprehensive data-model comparison of the transient evolution of global climate during the last 21,000 years; this comparison improved our understanding of global and regional climate changes and also raised new challenges to both models and proxy data.

\section{Background}

The large magnitude of climate change over the last 21 thousand years (kyr), documented by an extensive array of well-dated paleoclimate records, has made this period one of the best paleoclimate targets for testing climate-model estimates of climate sensitivity and the ability of models to simulate abrupt climate change. Model-data comparisons have remained a challenge, however, because model simulations of global climate are usually limited to hundreds of years while proxy records that span the entire interval are limited in their spatial coverage.

To address these issues, model-data comparisons have traditionally used the "snapshot" strategy in which data representing a specific time slice (e.g. $21 \mathrm{kyr}$ before present (BP), 6 kyr BP) are portrayed on a map for comparison to climate-model results for that time slice. This strategy greatly improved our understanding of global climate changes that are driven by external forcing to the coupled ocean-atmosphere system, notably orbital forcing, greenhouse gasses and ice sheets (COHMAP Members 1988), but it has several limitations.

From the data perspective, uncertainties in age models influence the map reconstruction, transferring uncertainties from the time domain to the space domain. From the perspective of mechanisms, while the snapshot strategy can be used to study the nearequilibrium surface responses, it cannot be used to study the response associated with the slow components of the climate system, such as the deep ocean, nor internal climate variability, such as the millennial-scale climate events and abrupt changes of the last deglaciation. The coarse temporal resolution between successive snapshots also makes it difficult for the snapshot approach to identify the complex temporal phasing relations between different climate events and thus assess mechanisms of climate change at regional and global scales.

Given these issues, the paleoclimate community recognized the importance of performing transient climate-model simulations that allow us to compare the results to the evolution of climate change recorded by data timeseries. In particular, such simulations should be conducted with synchronously coupled atmosphere and ocean components, as any asynchrony in the model, such as an acceleration in the forcing or a model component, will distort the response of the temporal evolution of the slow components, notably the deep ocean,

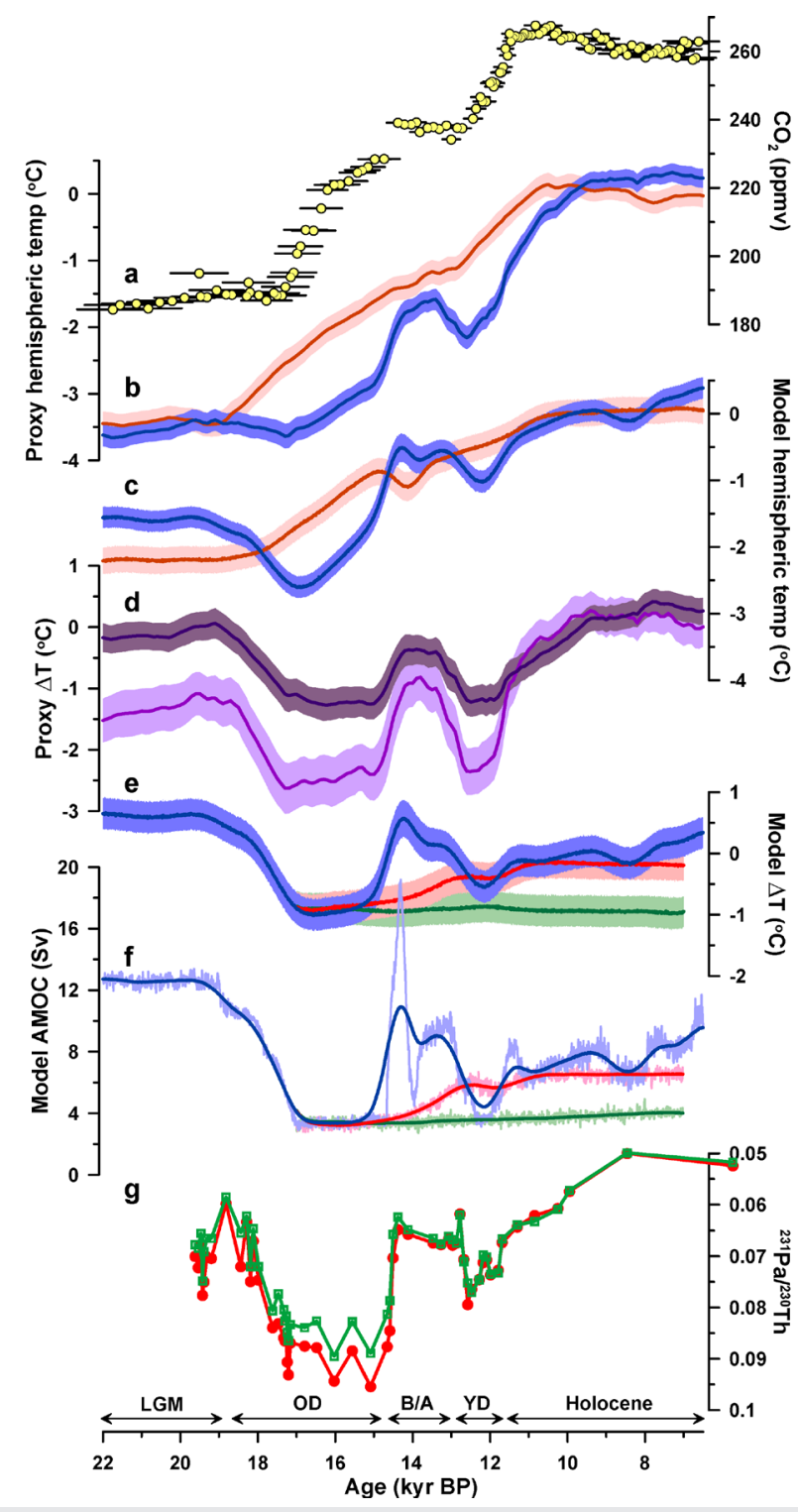

Figure 1: Hemispheric temperatures. (A) Atmospheric $\mathrm{CO}_{2}$ concentration. (B) Northern Hemisphere (blue) and Southern Hemisphere (red) proxy temperature stacks. (C) Modeled Northern Hemisphere (blue) and Southern Hemisphere (red) temperature stacks from the TRACE simulation. (D) Northern Hemisphere minus Southern Hemisphere proxy temperature stacks (dark purple). North Atlantic minus South Atlantic region proxy temperature stacks (light purple). (E) Modeled Northern Hemisphere minus Southern Hemisphere temperature stacks in the TRACE (blue), $\mathrm{CO}_{2}$ (red) and ORB (green) simulations. (F) Modeled AMOC strength in the ALL (blue), $\mathrm{CO}_{2}$ (red), and ORB (green) simulations. (G) North Atlantic sediment core OCE326-GGC5 ${ }^{231} \mathrm{~Pa} /{ }^{230} \mathrm{Th}$. Temperatures are given as deviations from the early Holocene (11.5-6.5 kyr BP) mean. Figure reproduced with permission from Shakun et al. (2012).

and can thus only be used approximately for the quasi-equilibrium response of surface ocean and the associated climate variability to external forcing.

The rapid advance in high performance computing over the last decade has now made it possible to simulate the transient climate evolution on multimillennial timescales in state-of-the-art, synchronously coupled ocean-atmosphere models. Here we summarize the SynTRACE-21 project, in which the Community Climate System Model 3 was used to simulate the transient climate evolution of the last 21,000 years (TRACE-21) and promote model-data comparison. The model has a 3.75-degree horizontal resolution for the atmosphere, a variable resolution 
from $\sim 3.6$ degrees at high latitude to $\sim 0.9$ degrees in the ocean (Yeager et al. 2006).

Supported jointly by PAGES, the US National Science Foundation, the US Department of Energy, the US National Center for Atmospheric Research, and Brown University, SynTRACE-21 was led by a steering committee of US-based modelers (Z. Liu, Univ. Wisconsin-Madison; B. Otto-Bliesner, National Center for Atmospheric Research) and data developers (P.U. Clark, Oregon State Univ.; J. Lynch-Stieglitz, Georgia Tech.; J. Russell, Brown University) and ultimately involved dozens of scientists around the world. After two US NSF-funded community workshops in Madison, WI (2008), and Boulder, CO (2009), two PAGES workshops were held: the first at Timberline Lodge, Mt. Hood, OR (9-13 October 2010), and the second in Providence, RI (3-7 November 2012), with several other meetings coordinated with other projects and conferences.

\section{SynTRACE-21 outcomes}

Using changing insolation, proxy data of greenhouse gas forcing, reconstructions of ice-sheet size and coastline, and an assumed history of freshwater water forcing in the North Atlantic, Liu et al. (2009) first simulated the transient climate evolution of the coupled atmosphere-ocean-terrestrial vegetation system for the last $21 \mathrm{kyr}$ in a baseline experiment (TRACE-21). This experiment, along with additional sensitivity experiments, was then used for comparison with data and for understanding the mechanism of the response.

\section{Global temperature changes}

The deglacial evolution of global climate from the Last Glacial Maximum (LGM, 21 kyr BP) to the early Holocene ( 11 kyr BP) presents an outstanding opportunity to combine TRACE-21 simulations with data to better understand the transient response of Earth's climate system to major climate forcing factors. The forcing factors include the changes of the external forcing associated with the Earth's orbit, the $\sim 80$ ppm rise of atmospheric greenhouse gases (GHG), as well as internal forcing of continental ice sheet and meltwater inputs to the ocean that result in changes in the Atlantic Meridional Overturning Circulation (AMOC). A major effort has been made by the paleoclimate research community to characterize these changes through the development and synthesis of well-dated, high-resolution records from the deep and intermediate ocean as well as from the continents, as summarized in Clark et al. (2012). The synthesis indicates that the superposition of two leading modes of climate change explains much of the variability in regional and global climate during the last deglaciation, with a strong association between the first mode and variations in greenhouse gases, and between the second mode and variations in the AMOC.

Shakun et al. (2012) further reconstructed the global surface temperature (largely seasurface temperature) from proxy records and compared the evolution of the reconstructed global and hemisphere mean temperatures (Fig. 1). They found that global surface temperature is correlated with and, furthermore,

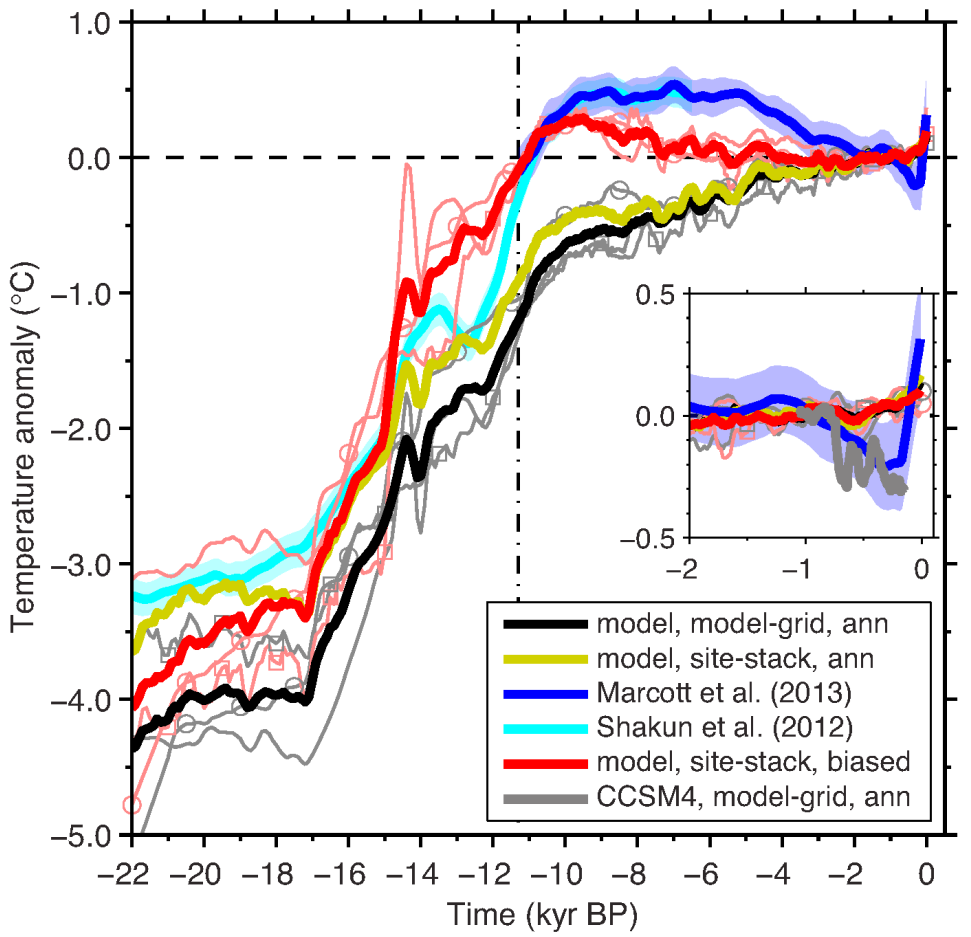

Figure 2: Evolution of the global surface temperature of the last 22,000 years: the reconstruction of Marcott et al. (2013) (blue) after $11.3 \mathrm{kyr}$ BP and Shakun et al. (2012) (cyan) before $6.5 \mathrm{kyr} \mathrm{BP,} \mathrm{the} \mathrm{model} \mathrm{annual} \mathrm{global}$ temperature averaged over the global grid points (black) and the model seasonally biased temperature averaged over the proxy sites (red). The models are CCSM3, FAMOUS, and LOVECLIM, with the ensemble mean in heavy, solid lines and individual member in light, thin lines (LOVECLIM and FAMOUS marked by circles and squares, respectively). Each temperature curve is aligned at $1 \mathrm{kyr} \mathrm{BP.} \mathrm{The} \mathrm{ensemble} \mathrm{mean} \mathrm{model}$ annual temperature averaged over proxy sites is also shown (yellow); its similarity to the model grid average demonstrates the insensitivity of the temperature trend to the average scheme. The insert shows the expanded part after $2 \mathrm{kyr}$ BP, with the addition of the last millennium experiment in CCSM4 (grey), which is forced additionally by volcanic aerosol and solar variability. Figure from Liu et al. (2014a). generally lags $\mathrm{CO}_{2}$ during the last deglaciation. The TRACE-21 simulation indicates that the large deglacial warming is caused by the large response of annual mean temperature to increasing GHGs, and the agreement with the data suggests comparable climate sensitivity in the model.

Differences between the respective temperature changes of the Northern and Southern Hemispheres parallel variations in the strength of the $\mathrm{AMOC}$ reconstructed from marine sediments. Consistent with the TRACE-21 simulations, these observations support the conclusion that an anti-phased hemispheric temperature response to the AMOC superimposed on globally in-phase warming driven by increasing $\mathrm{CO}_{2}$ concentrations can explain much of the temperature change during the last deglaciation (Fig. 1).

Marcott et al. (2013) extended the annual global surface temperature reconstruction through the Holocene ( 11-0 kyr BP; Fig. 2) The reconstruction showed that deglacial warming continued into the Holocene with temperatures plateauing in the early to mid-Holocene for global and hemispheric average temperatures, followed by a cooling of $\sim 1^{\circ} \mathrm{C}$ through the middle to late Holocene. This Holocene cooling trend in annual mean global temperature, however, is physically puzzling.

Under the dual forcing of a declining residual ice sheet and rising atmospheric $\mathrm{CO}_{2}$ transient climate-model simulations, including TRACE-21, exhibit a warming trend in the Holocene, in contrast to the reconstructed late-Holocene cooling in proxy data (Fig. 2; Liu et al. 2014a). The Holocene cooling trend in the data is more consistent with a response to summer insolation in the Northern Hemisphere and tropics, and thus may be attributed to a summer seasonal bias of the temperature, as simulated in models.

This potential summer bias, however, can't explain the data cooling trend in the Southern Hemisphere, potentially indicating model shortcomings in the representation of certain feedback processes. Overall, TRACE-21 has improved our understanding of the mechanism of major global climate changes and, furthermore, has stimulated studies on the potential biases both in the model and data interpretation (e.g. Marsicek et al. 2018).

\section{Regional hydroclimate changes}

Comparisons of TRACE-21 with terrestrial proxy data also provided insights into mechanisms of regional hydroclimate changes over the last 21,000 years. For example, Otto-Bliesner et al. (2014) studied climate change during the last deglaciation in Africa (Fig. 3). Proxy data show that wet conditions developed abruptly $\sim 14,700$ years ago in southeastern equatorial and northern Africa and continued into the Holocene. The abrupt onset and coherence of this early African Humid Period, however, has been challenging to understand, because changes in seasonal insolation forcing in the southern 

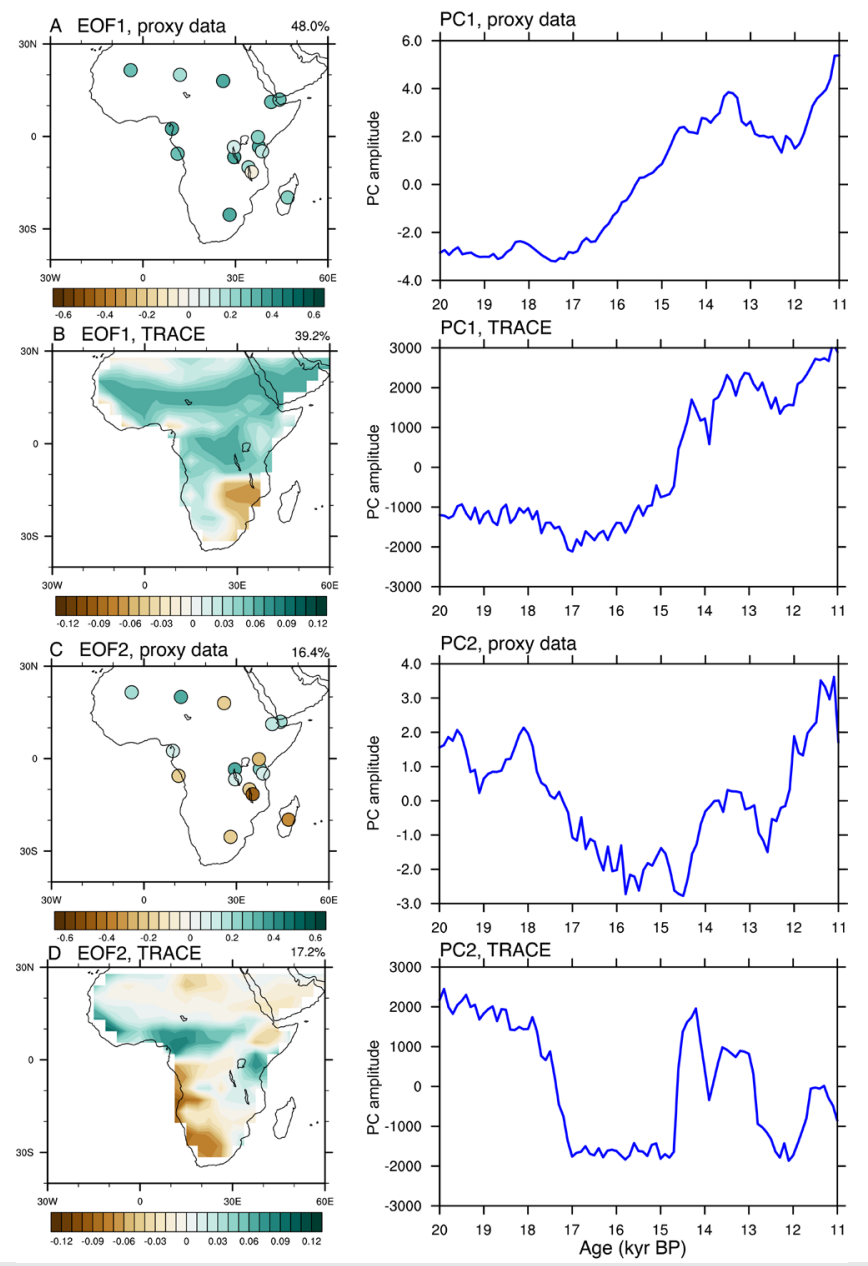

Figure 3: African hydroclimate for the deglacial period 20 to $11 \mathrm{kyr}$ BP. EOF1 and PC1 of (A) proxy data for moisture availability and (B) TRACE annual precipitation (millimeters per year). EOF1 explains $39.2 \%$ and $48.0 \%$ of the total variance of model precipitation and proxy data, respectively. (C) and (D) same as (A) and (B) except for EOF2 and PC2. EOF2 explains $17.2 \%$ and $16.2 \%$ of the total variance of model precipitation and proxy data, respectively. Model results and proxy records are interpolated to the same 100 -year resolution. Figure reproduced with permission from Otto-Bliesner et al. (2014).

tropics should weaken the austral monsoons (Otto-Bliesner et al. 2014).

Comparing the data with TRACE-21 simulations shows that a meltwater-induced reduction of the AMOC during the early deglaciation suppressed precipitation in both regions (Fig. 3). Once the AMOC was reestablished, wetter conditions developed north of the equator in response to high summer insolation and increasing GHG concentrations, whereas wetter conditions south of the equator were a response primarily to the GHG increase.

The TRACE-21 simulations have provided similar constraints for a number of other studies of regional precipitation. For example, Liu et al. (2014b) investigated the relationships between deglacial evolution of the East Asian Summer Monsoon (EASM) and oxygen isotope records from speleothems. The $\delta^{18} \mathrm{O}$ records document a series of isotopic changes that vary coherently across the Asian monsoon region. This change is difficult to interpret as a response to local precipitation, which tends to change at regional scales.

Comparing the data with TRACE-21 simulations shows reasonable agreement between the speleothem $\delta^{18} \mathrm{O}$ records and southerly monsoon winds, demonstrating that the data can record large-scale changes in the EASM. The subtropical monsoon circulation exhibits a continental-scale response due to global climate forcing associated with insolation and AMOC, as well as atmospheric teleconnections. The $\delta^{18} \mathrm{O}$ values, however, are altered by changes in the upstream source region, as well as local precipitation changes. Thus, despite the inherent computational limitations in model resolution and complexity, the TRACE-21 simulations provide insights into the paleoclimate proxies and large-scale monsoon dynamics.

\section{Perspective}

TRACE-21 has now been widely used by the paleoclimate community, ushering in a new era of seamless model-data comparison of transient climate evolution and abrupt climate changes from seasonal to orbital timescales, from regional to global spatial scales, and from the atmosphere to the deep ocean (e.g. Marsicek et al. 2018; Kaufman et al. 2020).

SynTRACE-21 has also built upon earlier data-model comparisons in demonstrating the effectiveness of this approach for improving our understanding of the mechanisms responsible for the climate evolution recorded by the data, as well as in identifying potential shortcomings in models and data. The model-data comparison of transient climate evolution has also stimulated further studies on the stability of the climate system, such as the AMOC, in the past, as well as for the future (Liu et al. 2017).

With the continued development of highperformance computing and improvements and increase in the number of proxy records, paleoclimate research will further benefit from new model-data studies beyond SynTRACE-21. First, for a direct comparison with the observed proxy variables and model variables, models need to be improved to include paleo proxy tracers, such as stable isotope ratios in foraminifera and other geochemical tracers (Brady et al. 2019). Second, model resolution should be improved so that detailed regional conditions at the location of the proxy data can be better simulated, e.g. IsoROMS (Stevenson et al. 2015) and the isotope-enabled model WRF (Moore et al. 2016).

One ultimate objective of combining data with models is the data assimilation of paleo proxies in advanced climate models, which requires further improvement of the estimation of the uncertainty of the proxy records as well as models (Tierney et al. 2020). These assimilation products will not only provide dynamically consistent reanalyses of the state of past climate, but may also help to constrain parameters and processes in future generations of Earth system models, thus further enhancing our ability to predict the future response of Earth's climate to GHG emissions.

\section{AFFILIATIONS}

'Department of Geography, Ohio State University, Columbus, USA

${ }^{2}$ National Center for Atmospheric Research, Boulder, CO, USA

${ }^{3}$ College of Earth, Ocean and Atmospheric Sciences, Oregon State University, Corvallis, USA

${ }^{4}$ School of Earth and Atmospheric Sciences, Georgia Institute of Technology, Atlanta, USA

${ }^{5}$ Department of Earth, Environmental and Planetary

Sciences, Brown University, Providence, RI, USA

\section{CONTACT}

Zhengyu Liu: liu.7022@osu.edu

\section{REFERENCES}

Brady E et al. (2019) J Adv Model Earth Syst 11: 2547-2566

Clark PU et al. (2012) Proc Natl Acad Sci USA 109: E1134-E1142

COHMAP Members (1988) Science 241: 1042-1052

Kaufman D et al. (2020) Sci Data 7: 201

Liu W et al. (2017) Sci Adv 3: e1601666

Liu Z et al. (2009) Science 325: 310-314

Liu Z et al. (2014a) Proc Natl Acad Sci USA 111:

$$
\text { E3501-E3505 }
$$

Liu Z et al. (2014b) Quat Sci Rev 83: 115-128

Marcott SA et al. (2013) Science 339: 1198-1201

Marsicek J et al. (2018) Nature 554: $92-96$

Moore M et al. (2016) J Geophys Res Atmos 121: 7235-7253

Otto-Bliesner BL et al. (2014) Science 346: 1223-1227

Shakun JD et al. (2012) Nature 484: 49-54

Stevenson S et al. (2015) Paleoceanogr Paleoclimatol 30: 1573-1593

Tierney JE et al. (2020) Nature 584: 569-573

Yeager SG et al. (2006) J Climate 19: 2545-2566 